Proc. XII Int. School on Theoretical Physics - Symmetry and Structural Properties of Condensed Matter

\title{
System Size Dependence of Entanglement in Quantum One-Dimensional Models
}

\author{
D. JAKUBCZYK ${ }^{a, *}$ AND P. JAKUBCZYK ${ }^{b}$ \\ ${ }^{a}$ Rzeszów University of Technology, al. Powstańców Warszawy 12, 35-959 Rzeszów, Poland \\ ${ }^{b}$ University of Rzeszów, T. Rejtana 16A, 35-959 Rzeszów, Poland
}

\begin{abstract}
We show that entanglement in one-dimensional spin and electron systems, with one excitation, depends only on the system size and has very simple form in both multipartite and bipartite case. Regarding the multipartite case, we present very simple expressions for global entanglement and $N$-concurrence, and show that they are mutually related. In the bipartite case, we give expressions for $I$-concurrence and negativity, and show that they are also dependent on each other.
\end{abstract}

DOI: 10.12693/APhysPolA.132.158

PACS/topics: 03.67.Bg, 03.67.Lx, 03.65.Ud

\section{Introduction}

The one-dimensional spin and electron systems are the subject of intense experimental research, one of the reason is because of their realisations in the lab in terms of ultracold atoms in an optical lattice, see inter alia $[1,2]$. Simultaneously, there is also unabated interest in quantum entanglement of spin qubits, see for example [3-6]. Therefore it seems that simple formulae which describe the entanglement in the case of states accessible in the lab may be very useful and allow experimentalists to develop their experimental intuition. One of the simplest spin states are those with one elementary excitation called one-magnon spin states. These states can be considered as generalization of $W$-states [7] because each single-node state $|j\rangle$ has a (local) phase factor $\mathrm{e}^{\mathrm{i} k j}$, where $k$ is the quasimomentum of the excitation - magnon for spin chain and $\mathrm{i}=\sqrt{-1}$. Therefore one expects that entanglement of the system prepared in one-excitation state is maximally persistent and robust under particle loss [8] and immune against global dephasing. This robustness may also lead to strong non-classicality [9] for large numbers of particles. Such states are desired elements in quantum information processing and quantum communication. Some one-excitation states of the electron systems have very similar properties and apply to the results obtained for spin systems.

The main aim of this paper is to show that in the spin system, which was prepared in one-magnon spin or electron state, quantum entanglement depends only on the sizes of the system and its appropriate subsystem and has very simple form. Moreover, we give analytical expressions for standard entanglement measures in multipartite (global entanglement, $N$-concurrence) and bipartite ( $I$ concurrence, negativity) case. We show that the results obtained for one-magnon spin states apply to the onedimensional electron systems with excitation provided by

*corresponding author; e-mail: djak@prz.edu.pl one electron or hole or electron pair that is two electrons of different spin projections.

The paper is organised as follows. Section 2 starts with a brief description of one-excitation spin and electron states as a generalization of $\mathrm{W}$ states. Next, in Sect. 3 we make survey of entanglement measures considered in the article. In Sect. 4 we present main results of this work, new entangled measure formulae applied to one- excitation spin or electron states. Conclusions, along with a brief summary of our results, can be found in Sect. 5.

\section{One-excitation spin and electron states}

Let us consider the Heisenberg XXX model consisting of $N$ qubits $\mathbb{C}^{2}$ on which one imposes periodic boundary conditions. The Hilbert space of such a system has the tensor product form $\mathcal{H}=\left(\mathbb{C}^{2}\right)^{\otimes N}$, with basis elements

$$
|f\rangle=\left|i_{1}\right\rangle \otimes\left|i_{2}\right\rangle \otimes \ldots \otimes\left|i_{N}\right\rangle, \quad i_{j} \in \tilde{2}, \quad j \in \tilde{N},
$$

where $\tilde{2}=\{0,1\}$ denotes single qubit basis states, and $\tilde{N}=\{1,2, \ldots, N\}$ is the set of all qubits, such that $\operatorname{dim} \mathcal{H}=2^{N}$. The Hamiltonian, which contains only exchange interactions between spins at neighbour sites, has the form

$$
\hat{H}=\frac{J}{2} \sum_{n=1}^{N}\left(P_{n, n+1}-I_{n, n+1}\right),
$$

with an effective parameter $J$. The operator $P_{n, n+1}$ exchanges two spin states at sites $n$ and $n+1$. In our case of the spin $s=1 / 2$ this operator can be represented in terms of the Pauli matrices $\boldsymbol{\sigma}=\left(\sigma^{1}, \sigma^{2}, \sigma^{3}\right)$ as

$$
P_{n, n+1}=1 / 2\left(\boldsymbol{\sigma}_{n} \boldsymbol{\sigma}_{n+1}+I_{n, n+1}\right)
$$

(the Dirac identity), $I_{n, n+1}$ denotes the identity matrix.

As one can notice (cf. Eq. (2)) the Heisenberg Hamiltonian is an algebra element of the symmetric group, therefore its action on magnetic configurations (1) does not change the number of qubits with spin down. Thus, the space $\mathcal{H}$ of all quantum states of the Heisenberg magnet decomposes as

$$
\mathcal{H}=\sum_{r=0}^{N} \oplus \mathcal{H}^{r},
$$


into invariant subspaces $\mathcal{H}^{r}, \operatorname{dim} \mathcal{H}^{r}=\left(\begin{array}{c}N \\ r\end{array}\right)$, with the fixed number $r$ of qubits with spins down (excitations). In this article we consider only states in the subspace $\mathcal{H}^{1}$ with one spin down. One can easily show, using Bethe ansatz [10], that eigenstates of the Hamiltonian (2) in this subspace are translationally covariant and represent magnon excitation, therefore we call them one-magnon spin states. These states, known in the literature as the basis of wavelets [11], have the Bloch form

$$
|\psi\rangle=\frac{1}{\sqrt{N}} \sum_{j=1}^{N} \mathrm{e}^{\mathrm{i} k j}|j\rangle,
$$

where, because of boundary periodic conditions, the wave number $k$ takes discrete values in the Brillouin zone $k=2 \pi n / N$, for $n=0,1, \ldots, N-1$. They can be interpreted as spin waves, with wave length $\lambda=2 \pi / k$ which periodically disturbs the ferromagnetic ground state with dispersion relation $E(k)=J(1-\cos k)$. In this picture the magnon can be treated a quasi-particle excitation from the ferromagnetic vacuum. In turn, $W$-states can be seen as homogeneous sum of a basis of orthonormal vectors in the Hilbert space of the system. In the case of Bethe ansatz for a quantum spin system, $W$-state is a descendent of the ferromagnetic vacuum under the shift operator $S^{-}$. A spin-wave state can be seen as a generalisation involving the phase $k$ between consecutive sites. Therefore, one-magnon spin states can be converted to the $N$-spin $W$-states by tuning local phases. This operation does not change the entanglement of the states (see, for example [12] for realization of spin system with trapped ions).

The one-dimensional Hubbard model of a finite chain of $N$ atoms has dynamics given by the Hubbard Hamiltonian in the following form:

$$
\begin{aligned}
\hat{H} & =-t \sum_{i \in\{+,-\}} \sum_{j \in \tilde{N}}\left(\hat{a}_{j, i}^{\dagger} \hat{a}_{j+1, i}+\hat{a}_{j+1, i}^{\dagger} \hat{a}_{j, i}\right) \\
& +U \sum_{j \in \tilde{N}} \hat{n}_{j+} \hat{n}_{j-},
\end{aligned}
$$

where $\tilde{N}$ denotes - similarly to the Heisenberg chain the set of nodes of the chain, $\hat{n}_{j, i}=\hat{a}_{j, i}^{\dagger} \hat{a}_{j, i}$, and finally $\hat{a}_{j, i}^{\dagger}, \hat{a}_{j, i}$ are the canonical Fermi operators, that is creation and anihilation operators of electron of spin $i$, on the site $j$. The Hilbert space $\mathcal{H}$ for $N_{e}$ electrons on $N$ nodes can be constructed as the tensor product of singlenode space $h_{j}$ of the form

$$
\mathcal{H}=\prod_{j=1}^{N} \otimes h_{j},
$$

with the basis of $h_{j}$ consisting of the vectors denoting all possible occupations of one node. Since we are dealing with fermions

$$
\operatorname{dim} h_{j}=4, h_{j}=l c_{\mathbb{C}}\{\emptyset,+,-, \pm\},
$$

where $\emptyset$ denotes the empty node, + and - stand for onenode spin projection equal to $1 / 2$ and $-1 / 2$ respectively, \pm denotes the double occupation of the one node by the two electrons with different spin projections, and $l c_{\mathbb{C}} A$ stands for the linear closure of a set $A$ over the field $\mathbb{C}$. One can obtain the final space $\mathcal{H}$ of all quantum states of the system also in the following way:

$$
\mathcal{H}=\sum_{N_{e}=0}^{2 N} \oplus \mathcal{H}^{N_{e}},
$$

where $\mathcal{H}^{N_{e}}$ denotes the space with fixed number of electrons $N_{e}$.

The one-excitation states of the Hubbard model considered in this paper are given by the formula (3), where $|j\rangle$ plays the role of the electron configuration. The electron configuration $f: \tilde{N} \longrightarrow \tilde{4}$, with $\tilde{4} \equiv\{\emptyset,+,-, \pm\}$, is the $N$-sequence of the elements from the set $\tilde{4}$ :

$$
\begin{aligned}
|f\rangle & =\left|i_{1}\right\rangle \otimes\left|i_{2}\right\rangle \otimes \ldots \otimes\left|i_{N}\right\rangle, \quad i_{j} \in \tilde{4}, \quad j \in \tilde{N}, \\
\text { with } & \\
\tilde{4}^{\tilde{N}} & =\{f: \tilde{N} \longrightarrow \tilde{4}\}, \mathcal{H}=l c_{\mathbb{C}} \tilde{4}^{\tilde{N}} .
\end{aligned}
$$

There are four possible cases of distribution electrons on the $N$-node chain resulting in the one-excitation states of the form (3). Since the Hubbard Hamiltonian has the obvious translational symmetry $\left(\hat{a}_{N+1, i}=\hat{a}_{1, i}\right)$ the basis of the appropriate Hilbert space must consist of only one orbit of the cyclic group. The cases are: only one electron, $N-1$ electrons of the same spin projection, $N+1$ electrons with $N$ of the same spin projection opposite to the remaining one and $2 N-1$ electrons case with $N$ electrons of the same spin projection opposite to left $N-1$ electrons. The appropriate eigenvalues can be written as $E(k)=-2 t \cos k$ with $k$ defined in Eq. (3).

\section{Entanglement measures}

In order to study entanglement we consider multipartite and bipartite case. In multipartite case we choose global entanglement and $N$-concurrence as a measures of entanglement while in bipartite case $I$-concurrence and negativity. These measures are computationally feasible and scalable quantification, they fulfil standard requirement for entanglement measures, in particular vanish for product states and are invariant under local unitary transformations.

Global entanglement $[13,14]$ is defined for a pure $N$ qubit state, it counts only bipartite correlations between each qubit and the rest of the system, and does not take into account true multipartite entanglement. To improve it, we use $N$-concurrence $[15,16]$, a generalization of $I$ concurrence to $N$-partite states. $N$-concurrence is defined for a pure normalized state and it allows us to calculate true multipartite entanglement.

$I$-concurrence is a generalization of the concurrence [17] (which is defined for a two-qubit system) to a system consisting of two subsystems $A$ and $B$ with an arbitrary dimensions. It is defined by closed expression only for pure states [18]. This quantity cannot exceed the value

$$
\sqrt{2\left(1-1 / d_{\min }\right)}
$$

where $d_{\min }$ is the lower of the dimensions of the two sub- 
systems $A$ or $B$. Negativity [19] is defined only for quantum bipartite systems $(A, B)$ of arbitrary dimensions described by density matrices. The maximum value of negativity depend on the dimensions of the subsystems.

\section{Entanglement caused by excitation}

As the number of qubits in the model increases then a study of entanglement measures described in Sect. 3 becomes a very hard computational problem and practically works only for models consisting of a more than a dozen of qubits. We remove this restriction for states with one excitation using the following observations. Firstly, one can show that if a system is prepared in the states (3) then entanglement does not depend on the wave number $k$ of the excitation - magnon in case of spin chain. Secondly, these states are translationally covariant with respect to discrete translations by any number of lattice spacings. Taking advantage of the above observations and definitions of entanglement (cf. [13-16, 18, 19]), we derived new very simple formulae for these measures.

Global entanglement for states chosen in Bloch form (3) depends only on the size of the system and has a form

$$
Q=4(N-1) / N^{2} .
$$

Formula (6) is not in fact new, because it was derived in [13] but here we quote it as an adequate measure. $N$ concurrence is $\sqrt{N^{3} /[8(N-1)]}$ times greater than global entanglement (for a given size $N$ of the system) and has a form

$$
N C=\sqrt{2-2 / N} .
$$

If the number of qubits tends to infinity, then $N$ concurrence and global entanglement are converging to $\sqrt{2}$ and 0 , respectively. Since, for big spin or electron rings prepared in state of the Bloch form (3) only the genuine $N$-partite entanglement remains, while bipartite entanglement disappears.

In order to describe disappearing of bipartite entanglement one decomposes the system into two parts of the lengths $\lambda_{1}, \lambda_{2}$, respectively. $\lambda_{1}$ denotes the number of qubits in the first and $\lambda_{2}$ in the second subsystem. The qubits are not necessarily contiguous. This division fulfils the obvious relations $\lambda_{1}, \lambda_{2} \in \mathbb{N}, \quad \lambda_{1} \leq \lambda_{2}, \quad \lambda_{1}+\lambda_{2}=$ $N$.

For the partition $\left(\lambda_{1}, N-\lambda_{1}\right)$ the $I$-concurrence can be calculated by means of the formula

$$
I C=2 \sqrt{\lambda_{1}\left(N-\lambda_{1}\right)} / N .
$$

The negativity for this partition is $2^{\lambda_{1}}-1$ times smaller and it has the form

$$
\mathcal{N}=2 \sqrt{\lambda_{1}\left(N-\lambda_{1}\right)} /\left[\left(2^{\lambda_{1}}-1\right) N\right] .
$$

As one can notice these bipartite measures depend only on the sizes of the system and its subsystem. It is also obvious that $I$-concurrence and negativity achieve maximum when $N=2 \lambda_{1}$ i.e. when dividing the system into two equal subsystems. Moreover, when the size of the system tends to infinity they both converge to zero whereas negativity achieves zero much faster, according to the factor 1/( $\left.2^{\lambda_{1}}-1\right)$ (cf. Eq. (9)).

\section{Conclusions and remarks}

We have shown that for the system prepared in the Bloch state of the form (3) considered entanglement measures depend only on sizes of the system and its subsystem. This easily allows one to prepare a system with desired amount of entanglement. Moreover, very tedious calculations of entanglement measures can be substantially simplified. Presented formulae allow one to calculate entanglement for an arbitrary size $N$, while the original definitions practically work only for the systems consisting of a few qubits. We expect that the size dependence of the entanglement of states with elementary excitation may help to understand entanglement in the systems with greater number of elementary excitations.

\section{References}

[1] M. Ganahl, E. Rabel, F.H.L. Essler, H.G. Evertz, Phys. Rev. Lett. 108, 077206 (2012).

[2] T. Fukuhara, P. Schauß, M. Endres, S. Hild, M. Cheneau, I. Bloch, C. Gross, Nature 502, 76 (2013).

[3] D. Das, H. Singh, T. Chakraborty, R.K. Gopal, C. Mitra, New J. Phys. 15, 013047 (2013).

[4] P. Jurcevic, B.P. Lanyon, P. Hauke, C. Hempel, P. Zoller, R. Blatt, C.F. Roos, Nature 511, 202 (2014).

[5] S. Sahling, G. Remenyi, C. Paulsen, P. Monceau, V. Saligrama, C. Marin, A. Revcolevschi, L. P. Regnault, S. Raymond, J.E. Lorenzo, Nature Phys. 11, 255 (2015).

[6] Y. Kravets, P. Jakubczyk, D. Jakubczyk, Phys. Rev. A 88, 022331 (2013).

[7] W. Dur, G. Vidal, J.I. Cirac, Phys. Rev. A 62, 062314 (2000).

[8] H.J. Briegel, R. Raussendorf, Phys. Rev. Lett. 86, 000910 (2001).

[9] A. Sen(De), U. Sen, M. Wieśniak, D. Kaszlikowski, M. Żukowski, Phys. Rev. A 68, 062306 (2003).

[10] H. Bethe, Z. Phys. 71, 205 (1931).

[11] B. Lulek, T. Lulek, A. Wal, P. Jakubczyk, Phys. B 337, 375 (2003).

[12] H. Haffner et al., Nature 438, 643 (2005).

[13] D.A. Meyer, N.R. Wallach, J. Math. Phys. 43, 4273 (2002).

[14] G.K. Brennen, arXiv:quant-ph/0305094, Quantum Inf. Comput. 3, 619 (2003).

[15] A.R.R. Carvalho, F. Mintert, A. Buchleitner, Phys. Rev. Lett. 93, 230501 (2004).

[16] F. Mintert, A.R.R. Carvalho, M. Kus, A. Buchleitner, Phys. Rep. 415, 207 (2005).

[17] W.K. Wootters, Phys. Rev. Lett. 80, 2245 (1998).

[18] P. Rungta, V. Bužek, C.M. Caves, M. Hillery, G.J. Milburn, Phys. Rev. A 64, 042315 (2001).

[19] G. Vidal, R.F. Werner, Phys. Rev. A 65, 032314 (2002). 